\title{
On the Asymptotic Stability of the Nonlinear Difference Equation System
}

\author{
Serbun Ufuk Değer ${ }^{*}$ and Yaşar Bolat ${ }^{1}$ \\ ${ }^{1}$ Department of Computer Programming, Kastamonu Vocational High School, Kastamonu University, Kastamonu, Turkey \\ ${ }^{2}$ Department of Mathematics, Faculty of Science and Arts, Kastamonu University, Kastamonu, Turkey \\ ${ }^{*}$ Corresponding author
}

\section{Article Info}

Keywords: Asymptotic stability, Difference equation, Liapunov stable. 2010 AMS: 39A10, 39A30.

Received: 13 March 2021

Accepted: 24 August 2021

Available online: 31 August 2021

\begin{abstract}
In this paper, we obtain some new results on the equi-boundedness of solutions and asymptotic stability for a class of nonlinear difference systems with variable delay of the form

$$
x(n+1)=a x(n)+B(n) F(x(n-m(n))), \quad n=0,1,2, \ldots
$$

where $F$ is the real valued vector function, $m: \mathbb{Z} \rightarrow \mathbb{Z}^{+}$, which is bounded function and maximum value of $m$ is $k$ and $B(n)$ is a $k \times k$ variable coefficient matrix. We carry out the proof of our results by using the Banach fixed point theorem and we use these results to determine the asymptotic stability conditions of an example.
\end{abstract}

\section{Introduction}

Nonlinear difference equations are the proper mathematical representation for discrete processes, which have remarkable importance in areas such as Nicholson's blowflies model, bobwhite quail population model and predator-prey models. Recently, many researchers have investigated asymptotic behaviour of the solutions of the nonlinear difference equations. Especially, Huong et al. $[5,6,9,10,11,12,13,14]$ have done important studies in this area in the last 20 years. For instance Giang-Huong $[5,6]$ obtained some new results for the asymptotic behaviour of solutions of nonlinear difference equations with time-invariant delay of the form

$$
x(n+1)=\lambda x(n)+F(x(n-m)) \quad n=0,1, \ldots,
$$

where $F:[0, \infty) \rightarrow[0, \infty)$ is a continuous function, $m \geq 0$ is a fixed integer and $\lambda \in(0,1)$. In addition, Huong [10] studied nonlinear difference equation with bounded multiple delay of the form

$$
x(n+1)=\lambda(n) x(n)+\sum_{i=1}^{r} \alpha_{i}(n) F(x(n-m(i))),
$$

where $n \in \mathbb{N}$ with $n \geq a$ for some $a \in \mathbb{N}$, where $r, m(i) \geq 1,1 \leq i \leq r$ are fixed positive integers, the functions $\alpha_{i}$ are defined on $\mathbb{N}$ and the functions $F$ are defined on $\mathbb{R}$. Also, Huong [11] obtained some oscillatory results for equation (1.1). Huong and Nam [12] investigated the oscillation, convergence and boundedness of solution of some nonlinear difference equations with multiple delay of the form

$$
x(n+1)=G\left(x(n), x\left(n-m_{1}\right), \ldots, x\left(n-m_{r}\right)\right) \quad n=0,1, \ldots,
$$

where $m_{i} \in \mathbb{N}_{0}, \forall i=1, \ldots, r$ and the function $G: \mathbb{R}^{m+1} \rightarrow \mathbb{R}$. After, Huong and Mau [9] studied on the stability of the zero of autonomous nonlinear difference equation with variable delay of the form

$$
x(n+1)=\lambda(n) x(n)+\alpha(n) F(x(n-m(n))) \quad n=0,1, \ldots
$$


where the functions $\lambda, \alpha$ are defined on the set of integers, the function $m$ maps the set of integers to the set of positive integers, the function $F$ is defined on the set of real numbers. Moreover, Huong [14] gave the some new results stability and strict boundedness conditions of non-autonomous nonlinear difference equation with time-varying delay of the form

$$
x(n+1)=\lambda(n) x(n)+\alpha(n) F(n, x(n-m(n))) \quad n=0,1, \ldots
$$

In addition to Huong's investigations, in the last century, there has been many literature on stability of delay difference equations. In particular, as examples of studies, Győri, Ladas and Vlahos [8], Chen and Liu [2], Graef and Qian [7], Edwards and Neville [4], Rao and Sudha [18], Asiri and Elsayed [19], Değer and Bolat [20], Muroya, Ishiwata and Guglielmi [16], Akgul, Inc and Karatas [22], Akgul [23], Zhang [21], Liao [15] can be given. In this study, we obtain some new results on the equi-boundedness of solutions and asymptotic stability for a class of nonlinear difference systems with variable delay of the form

$$
x(n+1)=a x(n)+B(n) F(x(n-m(n))), \quad n=0,1,2, \ldots
$$

where $F$ is the real valued vector function, $m: \mathbb{Z} \rightarrow \mathbb{Z}^{+}$, which is bounded function and maximum value of $m$ is $k$ and $B(n)$ is a $k \times k$ variable coefficient matrix. Let $\mathbb{Z}_{0}$ be as a set of integers belonging to the interval $\left[n_{0}-k, n_{0}\right]$ for each integer $n_{0} \geq 0$ (if $m$ is unbounded, then $\mathbb{Z}_{0}$ as a set of integers belonging to the interval $\left.\left(-\infty, n_{0}\right]\right)$. Also, let $\phi: \mathbb{Z}_{0} \rightarrow \mathbb{R}^{k}$ be an initial discrete bounded vector function. Now, we can give some definitions and proposition for the proof of the Lemma and Theorems.

Definition 1.1. If $x(n)=\phi(n)$ on $\mathbb{Z}_{0}$ and satisfies (1.2) for $n \geq n_{0}$, then $x(n)=x\left(n, n_{0}, \phi\right)$ is a solution of (1.2) [9].

Definition 1.2. If for any $\varepsilon>0$ and any integer $n_{0} \geq 0$ there exists a $\delta\left(n_{0}, \varepsilon\right)>0$ such that $\|\phi(n)\| \leq \delta$ on $\mathbb{Z}_{0}$ implies

$$
\left\|x\left(n, n_{0}, \phi\right)\right\| \leq \varepsilon \text { for } n \geq n_{0},
$$

then the zero solution of (1.2) is Liapunov stable [9].

Definition 1.3. If the zero solution of (1.2) is Liapunov stable and if for any integer $n_{0} \geq 0$ there exists $\mu\left(n_{0}\right)>0$ such that $\|\phi(n)\| \leq \mu\left(n_{0}\right)$ on $\mathbb{Z}_{0}$ implies

$$
\left\|x\left(n, n_{0}, \phi\right)\right\| \rightarrow 0 \text { as } n \rightarrow \infty,
$$

then the zero solution of (1.2) is asymptotically stable [9].

Definition 1.4. If there exists a $B\left(n_{0}, \phi\right)>0$ such that $\left\|x\left(n, n_{0}, \phi\right)\right\| \leq B\left(n_{0}, \phi\right)$ for $n \geq n_{0}$, then a solution $x(n)=x\left(n, n_{0}, \phi\right)$ of $(1.2)$ is said to be bounded [9].

Definition 1.5. If for any $n_{0}$ and any $B_{1}>0$ there exists $B_{2}=B_{2}\left(n_{0}, B_{1}\right)>0$ such that $\phi(n) \leq B_{1}$ on $\mathbb{Z}_{0}$ implies

$$
\left\|x\left(n, n_{0}, \phi\right)\right\| \leq B_{2} \text { for } n \geq n_{0},
$$

then solutions of (1.2) is equi-bounded [9].

Definition 1.6. A Banach space is a complete, normed, vector space.

Definition 1.7. Let $P: X \rightarrow X$ be a mapping from a set $X$ to itself. We call a point $x \in X$ a fixed point of $P$ if $P(x)=x[3]$.

Proposition 1.8. Let $X$ be a Banach space and $P: X \rightarrow X$ be a map such that

$$
\|P x-P y\| \leq \alpha\|x-y\|
$$

for some $0 \leq \alpha<1$ and all $x, y \in X$. Then $P$ has a unique fixed point in $X$. Moreover for any $x_{0} \in X$ the sequence of iterates $x_{0}, P x_{0}, P P x_{0}, \ldots$ converges to the fixed point of $P[3]$.

When $\|P x-P y\| \leq \alpha\|x-y\|$ for some $0 \leq \alpha<1$ and all $x, y \in X, P$ is called contraction. Also, the fact that $P$ is contraction implies the existence of a solution of equation (1.3). A contraction shrinks distances by a uniform factor $\alpha$ less than 1 for all pairs of points. Proposition 1.8 is called the contraction mapping theorem or Banach's fixed-point theorem. Also, for any sequence $\left(x_{k}\right)$, we denote $\sum_{k=a}^{b} x_{k}=0, \prod_{k=a}^{b} x_{k}=1$ for any $a>b$.

\section{Main Results}

Proposition 2.1. Suppose that $a \in(-1,1)-\{0\}$ and $B(n)$ is a nonsingular matrix function for all $n \in \mathbb{Z}$. Then $x(n)$ is a solution of system (1.2) if and only if

$$
x(n)=x\left(n_{0}\right) a^{n-n_{0}}+\sum_{t=n_{0}}^{n-1} B(t) F(x(t-m(t))) a^{n-t-1} .
$$


Proof. Consider the system (1.2). We multiply both sides of equation (1.2) by $\prod_{s=n_{0}}^{n} a^{-1}$

$$
\Delta\left(x(n) \prod_{s=n_{0}}^{n-1} a^{-1}\right)=B(n) F(x(n-m(n))) \prod_{s=n_{0}}^{n} a^{-1}
$$

is obtained. If equation (2.2) is summed from $n_{0}$ to $n-1$,

$$
\begin{aligned}
\sum_{t=n_{0}}^{n-1} \Delta\left(x(n) \prod_{s=n_{0}}^{n-1} a^{-1}\right) & =\sum_{t=n_{0}}^{n-1} B(n) F(x(n-m(n))) \prod_{s=n_{0}}^{t} a^{-1}, \\
x(n) \prod_{s=n_{0}}^{n-1} a^{-1}-x\left(n_{0}\right) & =\sum_{t=n_{0}}^{n-1} B(n) F(x(n-m(n))) \prod_{s=n_{0}}^{t} a^{-1}, \\
x(n) \prod_{s=n_{0}}^{n-1} a^{-1} & =x\left(n_{0}\right)+\sum_{t=n_{0}}^{n-1} B(n) F(x(n-m(n))) \prod_{s=n_{0}}^{n-1} a^{-1} .
\end{aligned}
$$

At last we have

$$
x(n)=x\left(n_{0}\right) \prod_{s=n_{0}}^{n-1} a+\sum_{t=n_{0}}^{n-1} B(n) F(x(n-m(n))) \prod_{s=t+1}^{n-1} a .
$$

The proof is completed.

Theorem 2.2. Suppose that the following conditions are satisfied:

(i) $F(0)=0$ and $F$ is locally Lipschitz in $x$. That is, there is a $M>0$ such that if $\|x\|,\|y\| \leq M$, then

$$
\|F(x)-F(y)\| \leq L\|x-y\|
$$

for positive constant $L$.

(ii) There exists $a \in(-1,1)-\{0\}, b \in(0,1)$ and $\|B(t)\|=\|B(t)\|_{1}$ such that

$$
L \sum_{t=n_{0}}^{n-1}\|B(t)\| \leq b
$$

for $n \geq n_{0}$. Then the solutions of (1.2) are equi-bounded.

Proof. Let $B_{1}$ be a positive constant. We choose $B_{2}>0$ such that $B_{1} \leq(1-b) B_{2}$. Also, let $\phi(n)$ be an initial discrete bounded function which satisfies $\|\phi(n)\| \leq B_{1}$ on $\mathbb{Z}_{0}$ of (1.2) for $n \geq n_{0}$. We define

$$
H=\left\{\mu: \mathbb{Z} \rightarrow \mathbb{R}^{k} \mid \mu(n)=\phi(n) \text { on } \mathbb{Z}_{0} \text { and }\|\mu\| \leq B_{2}\right\}
$$

where $\|\mu\|=\max _{n \in \mathbb{Z}}\|\mu(n)\|$. Firstly, we must show that $(H,\|\cdot\|)$ is a complete metric space. $(H,\|\cdot\|)$ is a metric space. It is clear that the metric space conditions are provided. Now we shall prove that every Cauchy sequence of points in $H$ has a limit that is also in $H$. Assume that $\left(\mu^{\beta}\right)$ is a Cauchy sequence in $H$. Then, we have

$$
\forall \varepsilon>0, \exists \beta_{0}: \forall \alpha, \beta \geq \beta_{0}:\left\|\mu^{\beta}-\mu^{\alpha}\right\|<\varepsilon,
$$

or

$$
\forall \varepsilon>0, \exists \beta_{0}: \forall \alpha, \beta \geq \beta_{0}: \max _{n \in \mathbb{Z}}\left\|\left(\mu^{\beta}-\mu^{\alpha}\right)(n)\right\|<\varepsilon,
$$

or

$$
\forall \varepsilon>0, \exists \beta_{0}: \forall \alpha, \beta \geq \beta_{0}:\left\|\left(\mu^{\beta}-\mu^{\alpha}\right)(n)\right\|<\varepsilon, \quad \forall n \in \mathbb{Z}
$$

$\left(\mu^{\beta}(n)\right)$ is a Cauchy sequence in $\mathbb{R}^{k}$ for a fixed $n \in \mathbb{Z}$. we know that $\mathbb{R}^{k}$ is a complete metric space and thus, we can write

$$
\mu(n)=\lim _{l \rightarrow \infty} \mu^{\beta}(n), \quad \exists \mu(n) \in \mathbb{R}^{k} .
$$

Since $\mu^{\beta} \in H$, we get $\mu^{\beta}(n)=\phi(n)$ on $\mathbb{Z}_{0}$. By (2.6), we know that

$$
\mu(n)=\phi(n) .
$$

Furthermore, we have $\|\mu\| \leq B_{2}$ with $\left\|\mu^{\beta}\right\| \leq B_{2}$. That is $\mu \in H$. We define a mapping $P: H \rightarrow H$ such that

$$
(P \mu)(n)=\phi(n) \text { on } \mathbb{Z}_{0}
$$


and

$$
(P \mu)(n)=\phi\left(n_{0}\right) a^{n-n_{0}}+\sum_{t=n_{0}}^{n-1} B(t) F(\mu(t-m(t))) a^{n-t-1} .
$$

Notice that $P$ maps from $H$ to itself. Indeed, the properties of norm

$$
\|(P \mu)(n)\|=\left\|\phi\left(n_{0}\right) a^{n-n_{0}}+\sum_{t=n_{0}}^{n-1} B(t) F(\mu(t-m(t))) a^{n-t-1}\right\|,
$$

we have

$$
\|(P \mu)(n)\| \leq\left\|\phi\left(n_{0}\right)\right\|\left|a^{n-n_{0}}\right|+\sum_{t=n_{0}}^{n-1}\|B(t)\|\left\|F(\mu(t-m(t))) a^{n-t-1}\right\| .
$$

By $\|\mu\| \leq B_{2},\|\mu(t-m(t))\| \leq B_{2}$. Hence, we can write

$$
\|F(\mu(t-m(t)))\| \leq L\|\mu(t-m(t))\| \leq L B_{2} .
$$

Then, from (2.9) and (2.10), we obtain

$$
\begin{aligned}
\|(P \mu)(n)\| & \leq B_{1}+B_{2} L\left\|\sum_{t=n_{0}}^{n-1} B(t) a^{n-t-1}\right\| \\
& \leq B_{1}+B_{2} L\left\|\sum_{t=n_{0}}^{n-1} B(t)\right\| \\
& \leq B_{1}+B_{2} b \leq B_{2} .
\end{aligned}
$$

So, we can say that $P$ maps from $H$ to $H$. Now, let $\mu, v \in H$, then we get

$$
\begin{aligned}
\|(P \mu)(n)-(P v)(n)\| & =\left\|\sum_{t=n_{0}}^{n-1} B(t) F(\mu) a^{n-t-1}-\sum_{t=n_{0}}^{n-1} B(t) F(v) a^{n-t-1}\right\| \\
& \leq L \sum_{t=n_{0}}^{n-1}\|B(t)\|\|\mu-v\|\left|a^{n-t-1}\right| \\
& \leq b\|\mu-v\| .
\end{aligned}
$$

Thus, we have shown that the mapping $P$ is a contraction under the supremum norm. According to the contraction mapping principle, $P$ has a unique fixed point $\mu^{*} \in H$. So, we have $\left(P \mu^{*}\right)(n)=\mu^{*}(n)$. By $n_{0} \in \mathbb{Z}_{0}$ and $\mu^{*} \in H$, we can write $\phi\left(n_{0}\right)=\mu^{*}\left(n_{0}\right)$. Hence

$$
\mu^{*}(n)=\phi\left(n_{0}\right) a^{n-n_{0}}+\sum_{t=n_{0}}^{n-1} B(t) F\left(\mu^{*}(t-m(t))\right) a^{n-t-1},
$$

that is, $\mu^{*} \in H$ is a solution of (1.2) and solutions of (1.2) is equi-bounded. The proof is completed.

Corollary 2.3. Suppose that the conditions of Theorem 2.1 are satisfied. Then the zero solution of (1.2) is Liapunov stable.

Proof. Let $\varepsilon>0$ be such that $b \varepsilon \in(0,1)$. If we choose $0<\delta \leq \varepsilon(1-b)$, then $\delta+b \varepsilon \leq \varepsilon$. Also, let $\phi(n)$ be an initial discrete bounded function which satisfies $\|\phi(n)\| \leq \delta$ on $\mathbb{Z}_{0}$ of (1.2) for $n \geq n_{0}$. We define

$$
H=\left\{\mu: \mathbb{Z} \rightarrow \mathbb{R}^{k} \mid \mu(n)=\phi(n) \text { on } \mathbb{Z}_{0} \text { and }\|\mu\| \leq \varepsilon\right\},
$$

where $\|\mu\|=\max _{n \in \mathbb{Z}}\|\mu(n)\|$. It can be shown easily that $(H,\|\|$.$) is a complete metric space similar to proof of Theorem 2.1. Let us define the$ mapping $P: H \rightarrow H$ as follows

$$
(P \mu)(n)=\phi\left(n_{0}\right) a^{n-n_{0}}+\sum_{t=n_{0}}^{n-1} B(t) F(\mu(t-m(t))) a^{n-t-1} .
$$

Also, by proof of Theorem 2.1, we know that $P$ is a contraction and $\|P \mu\| \leq \varepsilon$ for any $\mu \in H$. Hence, the zero solution of (1.2) is Liapunov stable. Therefore, the proof is completed.

Theorem 2.4. Suppose that the following conditions are satisfied:

(i) $F(0)=0$ and $F$ is locally Lipschitz in $x$. That is, there is a $M>0$ such that if $\|x\|,\|y\| \leq M$, then

$$
\|F(x)-F(y)\| \leq L\|x-y\|
$$

for positive constant $L$.

(ii) There exist $a \in(-1,1)-\{0\}, b \in(0,1)$ and $\|B(t)\|=\|B(t)\|_{1}$ such that

$$
L \sum_{t=n_{0}}^{n-1}\|B(t)\| \leq b
$$

for $n \geq n_{0}$.

(iii) $|n-m(n)| \rightarrow \infty$ as $n \rightarrow \infty$.

Then the zero solution of (1.2) is asymptotically stable. 
Proof. Let $\phi(n)$ be an initial discrete bounded function which satisfies $\|\phi(n)\| \leq \eta\left(n_{0}\right)$ on $\mathbb{Z}_{0}$ of $(1.2)$ for $n \geq n_{0}$. We define

$$
H^{\star}=\left\{\mu: \mathbb{Z} \rightarrow \mathbb{R}^{k} \mid \mu(n)=\phi(n) \text { on } \mathbb{Z}_{0},\|\mu\| \leq \varepsilon \text { and }\|\mu(n)\| \rightarrow 0 \text { as } n \rightarrow \infty\right\},
$$

where $\|\mu\|=\max _{n \in \mathbb{Z}}\|\mu(n)\|$. It can be easily shown that $\left(H^{\star},\|\|.\right)$ is a complete metric space similar to proof of Theorem 2.1 . Let us define the mapping $P: H^{\star} \rightarrow H^{\star}$ as follows

$$
(P \mu)(n)=\phi\left(n_{0}\right) a^{n-n_{0}}+\sum_{t=n_{0}}^{n-1} B(t) F(\mu(t-m(t))) a^{n-t-1} .
$$

From the proof of Theorem 2.1, $P$ is a contraction and it maps from $H$ to itself. For the asymptotic stability, we shall show that

$$
(P \mu)(n) \rightarrow 0 \text { as } n \rightarrow \infty \text {. }
$$

Now, since $|a|<1$,

$$
\prod_{s=n_{0}}^{n-1} a=a^{n-n_{0}} \rightarrow 0 \text { as } n \rightarrow \infty
$$

Thus, $\phi\left(n_{0}\right) a^{n-n_{0}} \rightarrow 0$. In that case, it will be enough to show that

$$
\sum_{t=n_{0}}^{n-1} B(t) F(\mu(t-m(t))) \rightarrow 0 \text { as } n \rightarrow \infty
$$

Let $\mu \in H^{\star}$, then $\|\mu(t-m(t))\| \leq \varepsilon$. Since $\mu(t-m(t)) \rightarrow 0$ as $n-m(n) \rightarrow \infty$, there exists an $\varepsilon_{1}>0$ and $n_{1}>0$ such that $\|\mu(t-m(t))\|<\varepsilon_{1}$ for $n>n_{1}$. Also, from (2.16), there exist an $n_{2}>n_{1}$ such that $\left|a^{n-n_{0}}\right| \leq \frac{\varepsilon_{1}}{b \varepsilon}$ for $n>n_{2}$. Hence, for all $n>n_{2}$

$$
\begin{aligned}
& \left\|\sum_{t=n_{0}}^{n-1} B(t) F(\mu(t-m(t))) a^{n-t-1}\right\| \leq \sum_{t=n_{0}}^{n-1}\left\|B(t) F(\mu(t-m(t))) a^{n-t-1}\right\|, \\
& \leq \sum_{t=n_{0}}^{n_{1}-1}\left\|B(t) F(\mu(t-m(t))) a^{n-t-1}\right\|+\sum_{t=n_{1}}^{n-1}\left\|B(t) F(\mu(t-m(t))) a^{n-t-1}\right\| \\
& \leq \quad L \sum_{t=n_{0}}^{n_{1}-1}\left\|B(t) \mu(t-m(t)) a^{n-t-1}\right\|+L \sum_{t=n_{1}}^{n-1}\left\|B(t) \mu(t-m(t)) a^{n-t-1}\right\| \\
& \leq \varepsilon L \sum_{t=n_{0}}^{n_{1}-1}\left\|B(t) a^{n-t-1}\right\|+\varepsilon_{1} L \sum_{t=n_{1}}^{n-1}\left\|B(t) a^{n-t-1}\right\| \\
& \leq \varepsilon L \sum_{t=n_{0}}^{n_{1}-1}\left\|B(t) a^{n-t-1}\right\|+\varepsilon_{1} L \sum_{t=n_{1}}^{n-1}\left\|B(t) a^{n-t-1}\right\| \\
& \leq \varepsilon L \sum_{t=n_{0}}^{n_{1}-1} \| B(t) \prod_{s=t+1}^{n-1} a \prod_{s=n_{1}}^{n-1} a \mid+\varepsilon_{1} b \\
& \leq \varepsilon L\left|\prod_{s=n_{1}}^{n-1} a \sum_{t=n_{0}}^{n_{1}-1}\|B(t)\| \prod_{s=t+1}^{n_{1}-1} a\right|+\varepsilon_{1} b \\
& \leq \varepsilon \varepsilon_{1}+\varepsilon_{1} b .
\end{aligned}
$$

This implies that (2.15) is occurs. According to the contraction mapping principle, $P$ has a unique fixed point which solves (1.2). The proof is completed.

Example 2.5. Consider difference equation system

$$
x(n+1)=\frac{1}{2} x(n)+B(n) F\left(x\left(n-\left[\left|\frac{n}{2}\right|\right]\right)\right), \quad n \geq 0,
$$

where $[||$.$] denotes greater integer function, a=\frac{1}{2}, F(x)=\left(\begin{array}{c}x_{1}^{2} \\ x_{2}^{2}\end{array}\right)$ for $x=\left(\begin{array}{c}x_{1} \\ x_{2}\end{array}\right), 2^{-2 n+4 m(n)}>1$ for $m(n)=\left[\left|\frac{n}{2}\right|\right]$ and

$$
B(n)=\left(\begin{array}{cc}
\frac{1+2^{-2 n+2 m(n)}-2^{-2 m(n)}}{2^{-m(n)}\left(1+2^{-2 n+2 m(n)}\right)} & \frac{2^{-m(n)}}{1+2^{-2 n+2 m(n)}} \\
\frac{3+4.2^{-2 n+2 m(n)}}{2^{2 m(n)+2}\left(1+2^{-2 n+2 m(n)}\right)} & \frac{1}{2^{2 m(n)+2}\left(1+2^{-2 n+2 m(n)}\right)}
\end{array}\right), \text { for } \forall n \in \mathbb{N} .
$$

then the zero solution of (2.17) is asymptotically stable. Indeed,

(i) there is a $L=1$ such that $\|x\|_{1}=\left|x_{1}\right|+\left|x_{2}\right| \leq 1$, then

$$
\begin{aligned}
\|F(x)\|_{1} & =\left|x_{1}^{2}\right|+\left|x_{2}^{2}\right|=\left|x_{1}\right|^{2}+\left|x_{2}\right|^{2} \\
& \leq\left(\left|x_{1}\right|+\left|x_{2}\right|\right)^{2} \\
& \leq\left|x_{1}\right|+\left|x_{2}\right|=\|x\|_{1}
\end{aligned}
$$




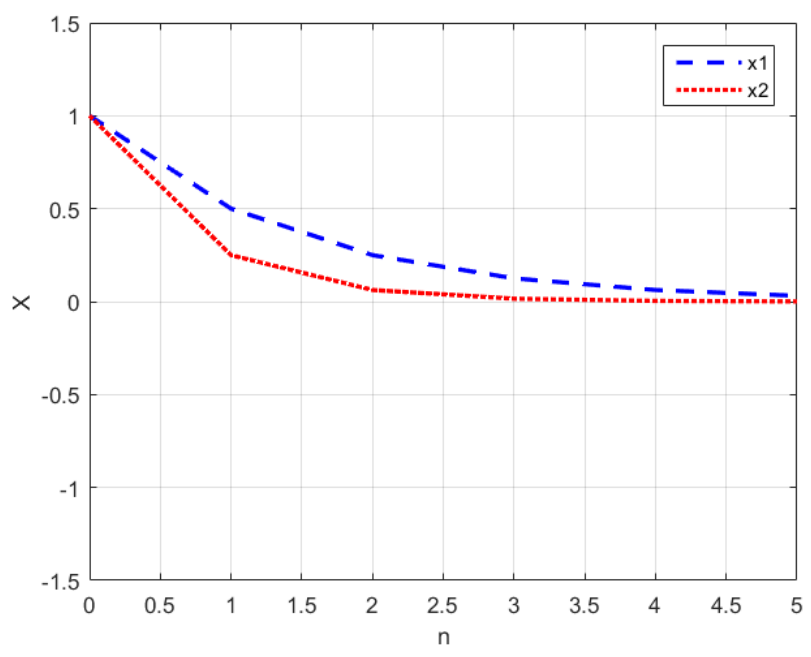

Figure 2.1: The behavior of the solution $x(n)=\left(\begin{array}{c}2^{-n} \\ 4^{-n}\end{array}\right)$ of system $(2.17)$

for $M=1$.

(ii) $\|B(n)\|_{1}=\max _{1 \leq i \leq n}\left(\sum_{j=1}^{n}\left|a_{i j}\right|\right)=\frac{1}{2^{m(n)}} \leq 1$

and $\lim _{n \rightarrow \infty} \frac{1}{2^{m(n)}}=0$. Furthermore, there exist $b \in(0,1)$ such that

$$
\sum_{t=n_{0}}^{n-1}\|B(t)\| \leq b
$$

for $n \geq n_{0}$.

(iii) $\left|n-\left[\left|\frac{n}{2}\right|\right]\right| \rightarrow \infty$ as $n \rightarrow \infty$.

Since the conditions of the theorem are provided, the system (2.17) is asymptotically stable. The behavior of the solutions of the system (2.17) is as Figure 1.

\section{Conclusion}

In this paper, we investigate the asymptotic stability of zero solution of system (1.2) which as an generalization of [9]. We used the Banach's fixed point theorem for the proof of the results. In this study, by using the definition of equi-bounded, initially, we showed that equation (1.2) is Liapunov stable after that we proved that this equation is asymptotically stable. Finally, we applied the these results to an example. As a result of this application we verified that equation (2.17) is suitable to the example

$$
x(n+1)=\frac{1}{2} x(n)+B(n) F\left(x\left(n-\left[\frac{n}{2}\right]\right)\right), \quad n \geq 0,
$$

where $F$ is the real valued vector function, $m: \mathbb{Z} \rightarrow \mathbb{Z}^{+}$, which is bounded function and maximum value of $m$ is $k$ and $B(n)$ is a $k \times k$ variable coefficient matrix.

\section{References}

[1] RP. Agarwal, Difference equations and inequalities: theory, methods, and applications CRC Press, (2000).

[2] MP. Chen, B. Liu, Asymptotic behavior of solutions of first order nonlinear delay difference equations Computers \& Mathematics with Applications, (1996), 32.4: 9-13.

[3] K. Conrad, The Contraction Mapping Theorem II, (2014).

[4] J.T. Edwards, J.F. Neville, Boundedness and stability of solutions to difference equations Journal of Computational and Applied Mathematics, (2002), 140.1-2: 275-289

[5] D.V. Giang, D.C. Huong, Extinction, Persistence and global stability in models of population growth Journal of mathematical analysis and applications, (2005), 308.1: 195-207.

[6] D.V. Giang, D.C. Huong, Nontrivial periodicity in discrete delay models of population growth Journal of mathematical analysis and applications, (2005), 305.1: 291-295

[7] J.R. Graef, C. Qian, Global stability in a nonlinear difference equation Journal of Difference Equations and Applications, (1999), 5.3: 251-270.

[8] I. Győri, G. Ladas, P.N. Vlahos, Global attractivity in a delay difference equation Nonlinear Analysis: Theory, Methods \& Applications, (1991), 17.5: 473-479.

[9] D.C. Huong, N.V. Mau, On a nonlinear difference equation with variable delay Demonstratio Mathematica, (2013), 46.1: 123-135.

[10] D.C. Huong, On the asymptotic behaviour of solutions of a nonlinear difference equation with bounded multiple delay Vietnam Journal of Mathematics, (2006), 34.2: 163-170.

[11] D.C. Huong, Oscillation for a Nonlinear Difference Equation Vietnam Journal of Mathematics, (2009), 37.4: 537-549.

[12] D.C. Huong, P.T. Nam, On Oscillation, Convergence and Boundedness of Solutions of Some Nonlinear Difference Equations with Multiple DelayVietnam Journal of Mathematics, (2008), 36.2: 151-160. 
[13] D.C. Huong, Persistence and global attractivity for a discretized version of a general model of glucose-insulin interaction Demonstratio Mathematica, (2016), 49.3: 302-318.

[14] D.C. Huong, Asymptotic Stability and Strict Boundedness for Non-autonomous Nonlinear Difference Equations with Time-varying Delay Vietnam Journal of Mathematics, (2016), 44.4: 789-800.

[15] M. Liao, Global asymptotic stability of a family of nonlinear difference equations. Discrete Dynamics in Nature and Society, (2013).

[16] Y. Muroya, E. Ishiwata, N. Guglielmi, Global stability for nonlinear difference equations with variable coefficients Journal of mathematical analysis and applications 334.1 (2007): 232-247.

[17] S.U. Deger, Y.Bolat, Stability conditions a class of linear delay difference systems Cogent Mathematics, (2017), 4.1:129445

[18] V. Sree Hari Rao, G.Jaya Sudha, Global Behaviour of Solutions of Nonlinear Delay Difference Equations. The Journal of Difference Equations and Applications, (2002), 8.2: 101-124.

[19] A. Asiri, E.M. Elsayed, Dynamics and Solutions of Some Recursive Sequences of Higher Order Journal of Computational Analysis\& Applications, (2019), 27.1.

[20] X. Zhang, J. Yan, Global asymptotic behavior of nonlinear difference equations Computers \& Mathematics with Applications, (2005), 49.9-10: $1335-1345$

[21] X. Zhang, Global attractivity for nonlinear difference equations with delay. Journal of Mathematical Analysis and Applications, (2007), 336 : 975-986.

[22] A. Akgul, M.Inc, E. Karatas Reproducing kernel functions for difference equations. Discrete and Continuous Dynamical Systems, (2015), 8(6).

[23] A. Akgul, On the solution of higher-order difference equationsy. Mathematical Methods in the Applied Sciences, (2015). 